Enzyme and Microbial Technology

Volume 120, January 2019, Pages 110-116

https://doi.org/10.1016/j.enzmictec.2018.10.005

\title{
THE ROLE OF ENZYME ADSORPTION IN THE ENZYMATIC DEGRADATION OF
}

AN ALIPHATIC POLYESTER

\author{
Péter Polyák ${ }^{1,2}$, Eszter Urbán ${ }^{1,2}$, Gergely N. Nagy ${ }^{3,4}$, Beáta G. Vértessy ${ }^{3,4}$, Béla \\ Pukánszky ${ }^{1,2 *}$
}

${ }^{1}$ Laboratory of Plastics and Rubber Technology, Department of Physical Chemistry and Materials Science, Budapest University of Technology and Economics, H-1521 Budapest, P.O. Box 91, Hungary

${ }^{2}$ Polymer Physics Research Group, Institute of Materials and Environmental Chemistry, Research Centre for Natural Sciences, Hungarian Academy of Sciences, H-1519 Budapest, P.O. Box 286, Hungary

${ }^{3}$ Department of Applied Biotechnology and Food Science, Budapest University of Technology and Economics, H-1521 Budapest, P.O. Box 91, Hungary

${ }^{4}$ Laboratory of Genome Metabolism and Repair, Institute of Enzymology, Research Centre for Natural Sciences, Hungarian Academy of Sciences, H-1519 Budapest, P.O. Box 286, Hungary

*Corresponding author: Tel: 36-1-463-2015, Fax: 36-1-463-3474, E-mail: bpukanszky@mail.bme.hu 


\section{ABSTRACT}

The enzyme catalyzed degradation of poly(3-hydroxybutyrate) (PHB) is a two-step process consisting of the adsorption of the enzyme on the surface of a PHB substrate and the cleavage of ester bonds. A deactivated enzyme was prepared by point mutagenesis to separate the two steps from each other. Measurements carried out with active and inactive enzymes on PHB particles proved that mutagenesis was successful and the modified enzyme did not catalyze degradation. Based on the Michaelis-Menten approach a kinetic model was proposed which could describe the processes quantitatively, the agreement between prediction and the measured data was excellent. The separation of the two processes allowed the determination of the adsorption kinetics of the enzyme; the rate constants of the adsorption and desorption process were determined for the first time. Comparison of these constants to reaction rates showed that adsorption is not instantaneous and can be the ratedetermining step. The area occupied by an enzyme molecule was also determined (13.1 $\left.\mathrm{nm}^{2}\right)$ and it was found to be smaller than the value published in the literature $\left(17 \pm 8 \mathrm{~nm}^{2}\right)$. The separation of the two steps makes possible the prediction and control of the degradation process.

Keywords: polyhydroxyalkanoates, enzymatic degradation, mutagenesis, adsorption kinetics, surface need 


\section{INTRODUCTION}

Biopolymers are used in increasing quantities in all areas of life. Their enzymatic degradation is of considerable interest in several applications, e.g. in their in vivo use as scaffolds, in the disposal of waste, during composting or in the production of various materials like biofuel or sugar. Recently the interest in controlled drug release also has increased enormously often requiring the degradation of the carrier matrix [1,2]. Since several enzymes have their optimum conditions close to, or even exactly at those prevailing in the human body [1-4], the enzyme catalyzed degradation of the carrier polymer is a convenient way to achieve controlled release. The approach has been used already to develop carrier matrices, which release the entrapped drug in this way [1-4].

The importance of enzymatically catalyzed reactions is increasing continuously. The vast majority of the currently applied enzyme reactions are carried out in a homogeneous media, usually in aqueous solution, thus the kinetics of the reactions can be described with conventionally applied models, like the Michaelis-Menten [5-8] or the Briggs-Haldane [913] approaches. However, if the reaction takes place under heterogeneous conditions, the models mentioned above cannot be applied, they either predict the outcome of the reaction with considerable systematic error, or even provide completely false or misleading results.

Although several technologies are applied also in practice using heterogeneous enzyme catalyzed reactions, appropriate models describing the kinetics of such reactions do not exist yet. In his attempt to create such a model, Kumar [14] introduced several modifications to the original model of Michaelis and Menten [15], with which he could describe quantitatively the kinetics of a number of autocatalytic reactions. Instead of a homogeneous reaction, Kumar [14] introduced two consecutive steps into the model, the adsorption of the enzyme and the actual catalysis. His model takes into account also the second or higher order kinetics of adsorption in the presence of two or multiple enzymes. 
Kari [16], on the other hand, focused on a single reaction, on the enzymatic degradation of cellulose and derived a model based on the original Michaelis-Menten kinetics. He considered the coverage of the surface by the enzyme as an important parameter influencing the kinetics of the reaction, thus he introduced an additional parameter into the equations describing the quasi steady state of the reaction. The modified model described the experimental data satisfactorily and the approach proved that the appropriate modification of the original Michaelis-Menten model could be applied even if the substrate is not soluble in water [16].

A number of groups working in the field of heterogeneous enzyme kinetics follow a different approach and develop semi-empirical or empirical models for the quantitative description of a specific heterogeneous substrate-enzyme reaction [17-19]. Diao [17], for example, studied the enzymatically catalyzed hydrolysis of a number of cellulose derivatives and estimated the time dependence of the average molecular weight of the degrading polymer phase by a particular solution of the differential equation describing first order kinetics. While Diao [17] ignored the formation and presence of different products, Kadam [19] developed a model based on the time dependent concentration of the most frequent metabolites forming during the enzymatically catalyzed degradation of lignocellulose [19]. His approach is entirely empirical, but rather successful, since it can predict values surprisingly close to those obtained by measurements [19].

The generation of a model, which describes the kinetics of heterogeneous enzyme reactions would require the separate analysis of all the steps taking place during heterogeneous enzymatic degradation. At least two steps must be considered during the analysis, the adsorption of the enzyme on the surface of the polymer and the actual catalysis. Consequently, these two steps must be separated for detailed kinetic characterization. This might be done, if one obtains enzyme molecules, which are able to adsorb on the surface of 
the polymer, but cannot initiate the reaction. Such a modification can be achieved by the inactivation of the catalytic site, while leaving intact the binding site responsible for the adsorption of the enzyme on the surface of the polymer. Such a substitution, i.e. a point mutation, can be achieved by the PCR (Polymerase Chain Reaction) site directed mutagenesis [20-24].

The goal of our work was to study and determine the adsorption kinetics of a hydrolase enzyme catalyzing the decomposition of PHB [25-28] independently of the chemical reaction itself. In order to achieve this goal, we used the method described above, i.e. PCR, to produce enzyme molecules, which adsorb on the surface of the polymer, but are inactive in catalysis. The kinetics of adsorption was followed by the measurement of the concentration of the enzyme in the medium surrounding the polymer. This procedure allows the determination of the total amount of enzyme required to cover completely the surface of the polymer, but also the surface need of an enzyme molecule [29]. A model is introduced which describes adsorption kinetics quantitatively. The calculation of rate constants allows the identification of the step determining the rate of the enzymatic degradation of aliphatic polyesters in heterogeneous reactions.

\section{EXPERIMENTAL}

\subsection{PCR mutagenesis}

Enzyme molecules carrying an inactive catalytic, and an intact binding site were prepared by the site-directed mutation of the intracellular enzyme natively synthetized by the strain Bacillus megaterium (ATCC 11561). The gene of the enzyme was isolated, characterized, and kindly supplied by Chen [30] in a pGS1941 plasmid. The plasmid was extracted from the carrier E. coli Origami (DE3) strain (Novagen) by using a NucleoSpin Plasmid Miniprep (Macherey-Nagel $\mathrm{GmbH}$ ). In the original gene sequence, the catalytic 
site (tgt, coding cysteine, Cys) was changed to the gct (coding alanine, Ala) sequence. PCR mutagenesis was carried out in a Thermo Hybrid PCR Spin (OyaGen) device. The Phusion ${ }^{\circledR}$ Site-Directed Mutagenesis Kit (Thermo Fisher Scientific-Finnzymes) containing a Phusion ${ }^{\circledR}$ Hot Start II High-Fidelity polymerase enzyme was applied.

The product of the PCR was purified with methylated DNA digestion catalyzed by DpnI nuclease, and was subsequently transformed into an E. coli RosettaBL21 (DE3) strain. Enzyme molecules carrying the inactivated catalytic site were expressed by growing the transformed RosettaBL21 culture in LB medium at $37^{\circ} \mathrm{C}$, which was later treated with a 0.1 $\mathrm{mmol} / \mathrm{dm}^{3}$ IPTG (isopropyl $\beta$-D-1-thiogalactopyranoside) solution. Cells were collected, disrupted by sonication, and subsequently centrifuged at $10000 \mathrm{rpm}$ for $30 \mathrm{~min}$. The remaining supernatant was used for the purification of HIS-tagged enzyme molecules by affinity chromatography on a Ni-nitrilotriacetic acid (NTA) agarose column. In order to compare the characteristics of the enzyme molecules having an inactive catalytic site to the original enzyme molecules, the native enzyme was also expressed by using the method described above on an E. coli Origami (DE3) strain containing the original, pGS1941 plasmid.

\subsection{Preparation of PHB suspensions}

Poly(3-hydroxybutyrate) (PHB) granules were obtained from Metabolix Ltd. (Mirel M2100, $\geq 99.5 \%$ purity) with an approximate crystallinity of $\sim 60 \%$. An $5 \mathrm{~m} / \mathrm{m} \%$ solution of the polymer was prepared in chloroform (Molar Chemicals Ltd., 98 \%) and then it was precipitated in technical grade denatured ethanol (Molar Chemicals Ltd., 96 \%). The ethanol was intensively stirred during precipitation with an Ultra-Turrax (Type T-25, IKAWerke $\mathrm{GmbH}$ ) at the high rate of $24000 \mathrm{rpm}$ in order to obtain a suspension with large interface area. 
The polymer particles were separated by filtration, then washed first with distilled water, and then with the buffer used for the enzymatic degradation. This buffer consisted of an aqueous solution of Tris/ $\mathrm{HCl}\left(10 \mathrm{mmol} / \mathrm{dm}^{3}\right)$ and $\mathrm{NaCl}\left(100 \mathrm{mmol} / \mathrm{dm}^{3}\right)$, both components supplied by Fluka $\mathrm{GmbH}$. The buffer also contained the co-factor of the enzyme $\left(\mathrm{Ca}^{2+}\right.$, applied in a form of $\mathrm{CaCl}_{2}$ salt, purchased from Sigma-Aldrich) in a concentration of $5 \mathrm{mmol} / \mathrm{dm}^{3}$, and it had a $\mathrm{pH}$ value of 8.0 .

The washing steps were followed by the re-suspension of the polymer particles in the buffer of the enzymatically catalyzed degradation. The suspension was stirred with an Ultra-Turrax at $6000 \mathrm{rpm}$ in order to hinder the aggregation of the polymer particles and to achieve the largest possible water-polymer interface.

\subsection{Degradation}

The degradation of the polymer was carried out in the presence of the original enzyme and the one modified by site-directed mutagenesis, respectively. Both enzymes were added to the polymer suspensions $\left(37^{\circ} \mathrm{C}, 200 \mathrm{rpm}\right)$ at the concentration of $7 \mu \mathrm{g} / \mathrm{ml}$ $\left(0.184 \mu \mathrm{mol} / \mathrm{dm}^{3}\right)$. Twelve samples were taken from the suspensions after different time intervals. The polymer particles were separated from the suspension in a centrifuge at 10000 rpm to avoid biasing the measurement of enzyme concentrations.

The clear supernatant obtained was analyzed by high performance liquid chromatography (HPLC) (Merck-Hitachi LaChrome Elite, equipped with a DAD detector). Chromatograms were recorded in the $200-350 \mathrm{~nm}$ wavelength range at a scanning period of 400 ms. The HPLC column (LiChroChart 250-4) contained a LiChrospher 100 RP-18 type end-capped silica (5 $\mu \mathrm{m}$ average particle size), the eluent used was a $10 \mathrm{mmol} / \mathrm{dm}^{3}$ phosphate buffer with the $\mathrm{pH}$ of 3.0 .

Besides the measurement of the concertation of the degradation products by HPLC, 
the size of the dispersed PHB particles was also determined in a suspension not containing any enzyme and in one subjected to degradation for 120 min catalyzed by either the native or the inactivated enzyme. The size distribution of PHB particles was determined by using a HORIBA Partica LA 950 A2 type apparatus.

\section{RESULTS AND DISCUSSION}

The results are presented in several sections. The effect and efficiency of point mutagenesis is analyzed in the first, followed by the presentation of the adsorption kinetics of the enzyme. A model describing kinetics quantitatively is introduced in the next section, while considerations related to the surface need of an enzyme molecule are presented in the final section of the paper.

\subsection{Effect of mutagenesis}

The efficiency of the site-directed mutagenesis was checked indirectly by the determination of degradation products in the aqueous media surrounding the polymer. Since the depolymerase enzyme Bacillus megaterium was shown to produce exclusively the monomer, 3-hydroxybutyric acid during degradation [30], the qualitative analysis of the metabolites is relatively simple. However, 3-hydroxybutyric acid must be separated from the other UV-absorbing components present in the buffer (tris and molecules of the depolymerase enzyme) that is achieved by the application of an eluent with the $\mathrm{pH}$ value of 3.0, since under acidic conditions the acid $\left(\mathrm{pK}_{\mathrm{a}}=4.7\right)$ is in the protonated form, its retention time is relatively long. The ionic molecules of tris and the enzyme are expected to have a retention time corresponding to the $\mathrm{t}_{0}$ value of the column.

The chromatograms of the degradation solutions containing the original, active 
enzyme are presented in Fig. 1 (a). According to the considerations presented above, two peaks can be observed in the chromatograms shown, one at $t_{0}$ and one at the retention time of the degradation product, respectively. The appearance of the peak assigned to the elution of 3-hydroxybutyric acid prove that the active enzyme catalyzes the degradation of PHB indeed, and changes in the area under the peak can be used for the quantitative analysis of degradation kinetics. The chromatograms recorded on solutions containing the modified enzyme are shown in Fig. 1 (b). The absence of the retention peak of 3-hydroxybutyric acid proves that mutagenesis was successful and the inactivated enzyme does not catalyze the depolymerization of PHB.

Another way to check the activity of the enzymes used is the measurement of the size of the dispersed polymer particles. The size distribution of the original particles, and those obtained in the presence of the active and inactive enzyme, respectively, after 120 hours of reaction are presented in Fig. 2. The figure clearly shows that the size distribution of the original particles and those extracted from the solution containing the inactive enzyme are identical. However, particle size is considerably smaller in the suspension containing the active enzyme indicating that degradation took place in the suspension in this case. The difference in particle size confirms again that mutagenesis was successful and further evaluation of the recorded chromatograms allows the quantitative analysis of adsorption kinetics.

\subsection{Adsorption kinetics}

The concentration of the enzyme in the solution must be measured in order to follow its adsorption kinetics on the surface of the PHB particles. However, the enzyme is in the ionized form in the suspension, thus its retention time equals to the $t_{0}$ of the column. On the other hand, it contains aromatic groups, e.g. tryptophan, which absorbs at around $260 \mathrm{~nm}$, 
thus enzyme concentration can be determined by UV spectroscopy. The spectra recorded on suspensions after different adsorption times are presented in Fig. 3. The maximum of the absorbance is around $250 \mathrm{~nm}$ instead of the usual 260-280 $\mathrm{nm}$. However, absorbance depends on the chemical environment of the molecule as well as on the structure of the enzyme itself, which may explain the shift. The quantitative analysis of enzyme adsorption was carried out at this wavelength to improve the accuracy of the measurement. Chromatograms recorded with detection at $250 \mathrm{~nm}$ are presented as a function of the time of adsorption in Fig. 4. The figure clearly shows that the concentration of the inactive enzyme decreases with increasing time, i.e. enzyme adsorption can be followed by the approach indeed.

The amount of both the active and the inactive enzyme was determined in the suspension by the integration of chromatographic peaks and it is presented in Fig. 5. The amount of both enzymes decreases initially and then approaches a value representing chemical equilibrium, which depends on the type of the enzyme. Decreasing concentration of the modified enzyme indicates that it retained its capability of absorbing on the surface of the polymer, an important observation since enzymes are very sensitive to any change in their primary structure. The difference in the equilibrium concentrations of the two enzymes results from the catalytic activity of the original enzyme. When it catalyzes the degradation reaction, the active complex decomposes; the enzyme leaves the surface and transfers into the suspension. Accordingly, catalysis results in the desorption of the enzyme from the surface and increases its concentration in the suspension. The inactive molecule remains on the surface after adsorption, thus its concentration decreases continuously in the degradation medium.

Catalytic activity changes also the apparent rate of adsorption. The concentration of active and inactive enzymes is plotted against each other in Fig. 6. At the beginning of the 
experiment the concentration of the two enzymes are the same, but later the deviation depends on the rate of the reaction/adsorption processes. The apparent rate of adsorption is smaller for the active enzyme, since it desorbs from the surface after each successful catalysis and its concentration is larger in the suspension than that of the inactive enzyme. Since in the case of inactive enzyme molecules catalysis is not possible, they either can be adsorbed on the surface forming an enzyme-substrate complex (abbreviated as ES) or located in the aqueous media. Consequently, the number of $E S$ complexes can be calculated by simply subtracting the measured amount of enzyme still present in the aqueous media (abbreviated as $E$ ) from their initial concentration $\left(0.184 \mu \mathrm{mol} / \mathrm{dm}^{3}\right)$. The two quantities, i.e. enzyme concentration in the suspension and the calculated amount of adsorbed molecules, are plotted against time in Fig. 7. The determination of the time dependence of the $[E S]$ and $[E]$ concentrations allows us the characterization of the kinetics of enzyme adsorption.

\subsection{The kinetic model}

Our approach is based on the Michaelis and Menten [15] model often used for the description of the kinetics of homogeneous enzymatic reactions. The model considers two steps, the formation of an enzyme-substrate complex, and then its subsequent dissociation, which results either in an unmodified substrate, or in the formation of a product molecule. Neither the former, nor the latter modifies the enzyme. The model can be formulated in the following way

$$
E+S \stackrel{k_{f}}{\rightleftharpoons} E S \stackrel{k_{c a t}}{\longrightarrow} E+P
$$

where $E$ indicates the enzyme, $S$ the substrate, $E S$ the activated complex, and $P$ the product; 
each step has its own rate constant $\left(k_{1}, k_{-1}, k_{c a t}\right)$. Accordingly, changes in the concentration of the enzyme can be expressed as

$$
\frac{d[E](t)}{d t}=-k_{f}[E](t)[S](t)+k_{r}[E S](t)+k_{c a t}[E S](t)
$$

and similarly the rate of the formation of the ES complex can be written as

$$
\frac{d[E S](t)}{d t}=k_{f}[E](t)[S](t)-k_{r}[E S](t)-k_{c a t}[E S](t)
$$

The concentration of the substrate decreases during the formation of the ES complex and increases in the dissociation of the latter when reaction does not occur

$$
\frac{d[S](t)}{d t}=-k_{f}[E](t)[S](t)+k_{r}[E S](t)
$$

while the product forms only during catalysis, i.e.

$$
\frac{d[P](t)}{d t}=k_{c a t}[E S](t)
$$

Although the Michaelis-Menten model described above is quite simple, calculations based on its differential equations (Eqs. 2-5) often provide surprisingly exact results. In our case, however, the model inevitably must be modified in order to account for the heterogeneous conditions and the inactivity of the modified enzyme. The substrates are ester groups in these experiments located at the surface of the PHB particles present in the 
suspension. Their number remains the same throughout the experiment, since the enzyme adsorbed on the surface cannot catalyze their hydrolysis. Consequently, the geometry of the suspended polymer particles does not change over time either. The constant number of ester groups is denoted by $S_{0}$ in subsequent treatment. Since the modified enzyme cannot catalyze the hydrolysis of $\mathrm{PHB}$, the rate of product formation is zero and also the concentration of the product is zero throughout the entire measurement. Taking into account these two modifications and considering that the concentration of two of the components, the substrate and the product, is independent of time, the equations become much simpler

$$
\begin{aligned}
& \frac{d[E](t)}{d t}=-k_{f}{ }^{\prime} \cdot[E](t)+k_{r} \cdot[E S](t) \\
& \frac{d[E S](t)}{d t}=+k_{f}^{\prime} \cdot[E](t)-k_{r} \cdot[E S](t)
\end{aligned}
$$

where the original rate constant for the formation of the ES complex $\left(k_{f}\right)$ is merged with the constant concentration of the substrate $\left(S_{0}\right)$ to give the modified constant, $\mathrm{kf}^{\prime}$.

Eqs. 6 and 7 form a linear, homogeneous, first order, autonomous differential equation system, which can be solved analytically. The solution takes the following form

$$
\begin{gathered}
{[E](t)=C_{1} \frac{k_{r}}{k_{f}^{\prime}}-C_{2} e^{-\left(k_{r}+k_{f}^{\prime}\right) t}} \\
{[E S](t)=C_{1}+C_{2} e^{-\left(k_{r}+k_{f}^{\prime}\right) t}}
\end{gathered}
$$

where $C_{1}$ and $C_{2}$ are integration constants the value of which can be determined by the fitting 
of the equations to the experimental data, while $k_{f}$ ' and $k_{r}$ are constants expressing the rate of adsorption and desorption, respectively. Fitting was done by the nonlinear iterative Levenberg-Marquardt algorithm and the functions obtained are indicated by solid lines in Fig. 7. The agreement between measurements and calculations is excellent, thus the kinetics of adsorption and desorption of the enzyme on the substrate can be described with the model with acceptable accuracy.

The rate constant $k_{f}^{\prime}$ and $k_{r}$ can be calculated from the integration constants, $C_{1}$ and $C_{2}$ and from the initial and boundary conditions. At $t=0$ the concentration of the enzyme was $7 \mu \mathrm{g} / \mathrm{ml}\left(0.184 \mu \mathrm{mol} / \mathrm{dm}^{3}\right)$, while that of the $E S$ complex zero. At $t=\infty$ the system is in dynamic equilibrium and the values can be obtained by extrapolation from the experimental data. $[E]\left(t_{\infty}\right)=2.29$ and $[E S]\left(t_{\infty}\right)=4.71 \mu \mathrm{g} / \mathrm{ml}$, respectively, were obtained for their value. Considering that at $t=\infty$, the exponential terms are infinitely small in Eqs. 8 and 9, we obtain the values of $4.71 \mu \mathrm{g} / \mathrm{ml}$, and $-4.71 \mu \mathrm{g} / \mathrm{ml}$ for $C_{1}$ and $C_{2}$, respectively. The knowledge of the integration constants allows us the calculation of the rate constants $k_{f}$ and $k_{r}$, for which $0.0519 \mathrm{~min}^{-1}$ and $0.0253 \mathrm{~min}^{-1}$, respectively, were obtained. The relation between these values, the rate of the adsorption being more than twice as large as the rate of the desorption shows that the chemical equilibrium is shifted towards adsorption. Adsorption is obviously thermodynamically favorable, thus in the state of equilibrium the majority of the enzyme molecules are adsorbed on the surface of the polymer. Although the values of $k_{f}$ and $k_{r}$ obtained apply exclusively to our conditions, enzyme and substrate, the approach can be generalized to other similar systems.

One must emphasize here that the rate of adsorption and desorption of an enzyme on a polyester substrate has never been determined before, thus we cannot compare the obtained values to published data. On the other hand, the rate of catalysis, i.e. $k_{c}$ values have been measured and published before (see Cornish [31], Cook [32], or Bisswanger [33]). The 
analysis of published data shows that the enzymes with the fastest catalysis rates falling in the range of $k_{c}=10^{1}-10^{6} \mathrm{~min}^{-1}$ are the metabolizers, followed by the restriction enzymes with a rate not significantly smaller than $k_{c}=10^{1} \mathrm{~min}^{-1}$. The slowest restriction enzymes are generally operating at a rate of $10^{-3} \mathrm{~min}^{-1}$. On this virtual scale, the slowest catalysts are the ligases attaching two molecules or groups to each other. Their rate is slower than the value of $10^{-3} \mathrm{~min}^{-1}$ mentioned above, and covers the range of $10^{-3}-10^{-6} \min ^{-1}$ [31-33]. The knowledge of the rate constant of the various enzymes offers the possibility to compare our calculated values directly to those published in the literature. The rate of adsorption of the enzyme natively produced by the strain Bacillus megaterium is significantly slower than the catalysis of the metabolizers, generally operating at a rate of $10^{1}-10^{6} \mathrm{~min}^{-1}$. Thus, we can say that the adsorption of our enzyme occurring at the calculated rate of $0.0519 \mathrm{~min}^{-1}$ is certainly not instantaneous; in fact, it is the rate-determining step in the enzymatic degradation of PHB.

\subsection{Surface need of an enzyme molecule}

The amount of enzyme molecules located on the surface of the PHB particles is given by the difference in their concentration at equilibrium (see Fig. 7) and at the start of the experiment $\left(0.184 \mu \mathrm{mol} / \mathrm{dm}^{3}\right)$. The surface area available for adsorption can be calculated from the number and the geometry of PHB particles present in the aqueous phase. As the latter would be difficult to obtain for individual particles, their shape is approximated with that of a sphere. The size distribution of the particles presented in Fig. 2 can be converted into surface as shown in Fig. 8.

The conversion of the size distribution into surface distribution and the integration of the function leads to the cumulated surface area of the particles present in the suspension. If the surface is completely covered by the enzyme, the surface need of one molecule can 
be calculated as the ratio of the area $\left(1.04 \cdot 10^{17} \mathrm{~nm}^{2}\right)$ and the total number of molecules adsorbed $\left(7.95 \cdot 10^{15}\right)$. Accordingly, the surface occupied by one enzyme molecule is 13.1 $\mathrm{nm}^{2}$. The surface need of the PHB depolymerase enzyme natively synthetized by Bacillus megaterium has not been published yet, but information exists for other, similar enzymes. According to Kasuya [29], for example, the extracellular depolymerase enzyme of Alcaligenes faecalis occupies a surface of $17 \pm 8 \mathrm{~nm}^{2}$, which is in the same range as the value obtained by us. The deviation might be explained by the fact that the catalytic domain of the enzyme applied by the Japanese researchers was not inactivated prior the measurement, thus their sample was far from complete coverage. The other factor, which must be taken into account, is the difference in the composition and structure of the enzymes. The extracellular enzyme from Alcaligenes faecalis possesses distinct domains for catalysis and binding linked by a linker domain. This is in contrast to the enzyme we have presently studied, in which the active site and the binding surface are located within one folded domain. The separate domain for binding in the extracellular enzyme may result in the larger surface need determined.

\section{CONCLUSIONS}

The enzyme catalyzed degradation of poly(3-hydroxybutyrate) is a two-step process consisting of the adsorption of the enzyme on the surface of PHB particles and the cleavage of ester bonds. A deactivated enzyme was prepared by point mutagenesis to separate the two steps from each other in order and study enzyme adsorption independently of catalysis. Measurements carried out with active and inactive enzymes proved that mutagenesis was successful and the modified enzyme did not catalyze degradation. Based on the MichaelisMenten approach, a kinetic model was proposed which could describe the processes quantitatively; the agreement between prediction and the measured data was excellent. The 
separation of the two processes allowed the determination of the kinetics of the adsorption of the enzyme. The rate constants of the adsorption and desorption process were determined for the first time. The comparison of these constant to reaction rates showed that adsorption is not instantaneous and it is the rate-determining step in the enzymatic degradation of PHB. The results obtained allowed the determination of the area occupied by one enzyme molecule to be $13.1 \mathrm{~nm}^{2}$. The separation of the two steps makes possible prediction and the control of the degradation process.

\section{ACKNOWLEDGEMENTS}

The National Research Fund of Hungary (OTKA K 120039) is greatly acknowledged for the financial support of this research, which was supported also by the National Research, Development and Innovation Office of Hungary [K119493, 2017-1.3.1VKE-2017-00013, VEKOP-2.3.2-16-2017-00013 and the BME-Biotechnology FIKP grant of EMMI (BME FIKP-BIO)].

\section{REFERENCES}

[1] M.V. Volokitina, V.A. Korzhikov-Vlakh, T.B. Tennikova, E.G. Korzhikova-Vlakh, Macroporous monoliths for biodegradation study of polymer particles considered as drug delivery systems, Journal of Pharmaceutical and Biomedical Analysis, 145 (2017) 169-177.

[2] I. Marchenko, A. Yashchenok, T. Borodina, T. Bukreeva, M. Konrad, H. Möhwald, A. Skirtach, Controlled enzyme-catalyzed degradation of polymeric capsules templated on CaCO3: Influence of the number of LbL layers, conditions of degradation, and disassembly of multicompartments, Journal of Controlled Release, 162 (2012) 599-605. 
[3] W.S.W. Shalaby, G.E. Peck, K. Park, Release of dextromethorphan hydrobromide from freeze-dried enzyme-degradable hydrogels, Journal of Controlled Release, 16 (1991) 355363.

[4] A.S. Bhalla, R.A. Siegel, Mechanistic studies of an autonomously pulsing hydrogel/enzyme system for rhythmic hormone delivery, Journal of Controlled Release, 196 (2014) 261-271.

[5] A. Cornish-Bowden, One hundred years of Michaelis-Menten kinetics, Perspectives in Science, 4 (2015) 3-9.

[6] A. Goldbeter, Oscillatory enzyme reactions and Michaelis-Menten kinetics, FEBS Letters, 587 (2013) 2778-2784.

[7] A. Cornish-Bowden, The origins of enzyme kinetics, FEBS Letters, 587 (2013) 27252730.

[8] G.L. Atkins, I.A. Nimmo, Current trends in the estimation of Michaelis-Menten parameters, Analytical Biochemistry, 104 (1980) 1-9.

[9] A.R. Tzafriri, E.R. Edelman, The total quasi-steady-state approximation is valid for reversible enzyme kinetics, Journal of Theoretical Biology, 226 (2004) 303-313. 
[10] H.S. Lee, J. Hong, Kinetics of glucose isomerization to fructose by immobilized glucose isomerase: anomeric reactivity of d-glucose in kinetic model, Journal of Biotechnology, 84 (2000) 145-153.

[11] A. Converti, M. Del Borghi, Simultaneous effects of immobilization and substrate protection on the thermodynamics of glucose isomerase activity and inactivation, Enzyme and Microbial Technology, 21 (1997) 511-517.

[12] S.R. Coy, R.D. Tanner, I. Endo, I. Inoue, Relating the hysteresis between the product and cell growth rates in fermentation processes by an analytical expression, Journal of Molecular Catalysis, 12 (1981) 375-383.

[13] G.A. Pinto, P.W. Tardioli, R.Y. Cabrera-Padilla, C.M.A. Galvão, R.C. Giordano, R.L.C. Giordano, Amino acids yields during proteolysis catalyzed by carboxypeptidase A are strongly dependent on substrate pre-hydrolysis, Biochemical Engineering Journal, 39 (2008) 328-337.

[14] J. Kumar, S. Nath, Analysis of autocatalytic reactions with Michaelis-Menten kinetics in an isothermal continuous stirred tank reactor, Chemical Engineering Science, 52 (1997) $3455-3462$

[15] K.A. Johnson, R.S. Goody, The Original Michaelis Constant: Translation of the 1913 Michaelis-Menten Paper, Biochemistry, 50 (2011) 8264-8269. 
[16] J. Kari, M. Andersen, K. Borch, P. Westh, An Inverse Michaelis-Menten Approach for Interfacial Enzyme Kinetics, ACS Catalysis, 7 (2017) 4904-4914.

[17] Y. Diao, M. Song, Y. Zhang, L.-y. Shi, Y. Lv, R. Ran, Enzymic degradation of hydroxyethyl cellulose and analysis of the substitution pattern along the polysaccharide chain, Carbohydrate Polymers, 169 (2017) 92-100.

[18] M. Wojtusik, M. Zurita, J.C. Villar, M. Ladero, F. Garcia-Ochoa, Influence of fluid dynamic conditions on enzymatic hydrolysis of lignocellulosic biomass: Effect of mass transfer rate, Bioresource Technology, 216 (2016) 28-35.

[19] K.L. Kadam, E.C. Rydholm, J.D. McMillan, Development and Validation of a Kinetic Model for Enzymatic Saccharification of Lignocellulosic Biomass, Biotechnology Progress, 20 (2004) 698-705.

[20] S. Simionatto, S.B. Marchioro, V. Galli, T.D. Luerce, D.D. Hartwig, Â.N. Moreira, O.A. Dellagostin, Efficient site-directed mutagenesis using an overlap extension-PCR method for expressing Mycoplasma hyopneumoniae genes in Escherichia coli, Journal of Microbiological Methods, 79 (2009) 101-105.

[21] Y. Kato, N. Arakawa, Y. Masuishi, H. Kawasaki, H. Hirano, Mutagenesis of longer inserts by the ligation of two PCR fragments amplified with a mutation primer, Journal of Bioscience and Bioengineering, 107 (2009) 95-97. 
[22] K.P. Kim, S.S. Cho, K.K. Lee, M.H. Youn, S.-T. Kwon, Improved thermostability and PCR efficiency of Thermococcus celericrescens DNA polymerase via site-directed mutagenesis, Journal of Biotechnology, 155 (2011) 156-163.

[23] Y. Adachi, C. Fukuhara, TA strategy for rapid and efficient site-directed mutagenesis, Analytical Biochemistry, 431 (2012) 66-68.

[24] K.B. Mullis, F.A. Faloona, 9 - Specific Synthesis of DNA in Vitro via a PolymeraseCatalyzed Chain Reaction A2 - Wu, Ray, Academic Press, San Diego, 1989.

[25] F. Li, C. Zhang, Y. Liu, D. Liu, H. Xia, S. Chen, Efficient production of (R)-3hydroxybutyric acid by Pseudomonas sp. DS1001a and its extracellular poly(3hydroxybutyrate) depolymerase, Process Biochemistry, 51 (2016) 369-373.

[26] J.M. Merrick, R. Steger, D. Dombroski, Hydrolysis of native poly(hydroxybutyrate) granules (PHB), crystalline PHB, and artificial amorphous PHB granules by intracellular and extracellular depolymerases, International Journal of Biological Macromolecules, 25 (1999) 129-134.

[27] H.-W. Jung, M.-K. Yang, R.-C. Su, Purification, characterization, and gene cloning of an Aspergillus fumigatus polyhydroxybutyrate depolymerase used for degradation of polyhydroxybutyrate, polyethylene succinate, and polybutylene succinate, Polymer Degradation and Stability, 154 (2018) 186-194. 
[28] T. Hiraishi, N. Komiya, M. Maeda, Y443F mutation in the substrate-binding domain of extracellular PHB depolymerase enhances its PHB adsorption and disruption abilities, Polymer Degradation and Stability, 95 (2010) 1370-1374.

[29] K.-i. Kasuya, Y. Inoue, Y. Doi, Adsorption kinetics of bacterial PHB depolymerase on the surface of polyhydroxyalkanoate films, International Journal of Biological Macromolecules, 19 (1996) 35-40.

[30] H.J. Chen, S.C. Pan, G.C. Shaw, Identification and Characterization of a Novel Intracellular Poly(3-Hydroxybutyrate) Depolymerase from Bacillus megaterium, Applied and Environmental Microbiology, 75 (2009) 5290-5299.

[31] A. Cornish-Bowden, Fundamentals of Enzyme Kinetics, Wiley-Blackwell, Berlin, 1979.

[32] P. Cook, W.W. Cleland, Enzyme Kinetics and Mechanism, Garland Science, London, 2007.

[33] H. Bisswanger, Enzyme Kinetics: Principles and Methods, Wiley-VCH, Weinheim, 2008. 


\section{CAPTIONS}

Fig. 1 HPLC chromatograms recorded on degradation suspensions that contained active (a) and inactive enzyme (b). A lack of the peak of the monomer (at 6 minutes) in case of inactive enzymes proves that the mutagenesis was successful.

Fig. 2 Size distribution of PHB particles in the degradation medium. Symbols: (solid) initial suspension, (dashed) suspension containing the inactive enzyme, (short dot) suspension containing the active enzyme.

Fig. 3 UV spectra recorded on the product appearing in the HPLC chromatogram at $t=2.1$ min retention time. Changing enzyme concentration with increasing time.

Fig. 4 Changing intensity of the chromatographic peak of the enzyme with increasing adsorption time.

Fig. 5 Dependence of the amount of the enzymes (peak area) in the suspension on the time of adsorption. Symbols: $(\square)$ active enzyme, $(\bigcirc)$ inactive enzyme.

Fig. 6 Difference in the rate of adsorption of the active and inactive enzyme. The active enzyme apparently adsorbs in smaller amounts than the inactive during the same period.

Fig. 7 Dependence of the concentration of the components on adsorption time. Symbols: $(\square)$ enzyme, $(\bigcirc)$ enzyme-substrate complex. The solid lines are functions predicted by the kinetic model proposed.

Fig. 8 Differential and cumulative surface area distributions of PHB particles suspended in the adsorption medium. Symbols: (solid) differential curve, (dashed) integral curve 
Polyák, Fig. 1

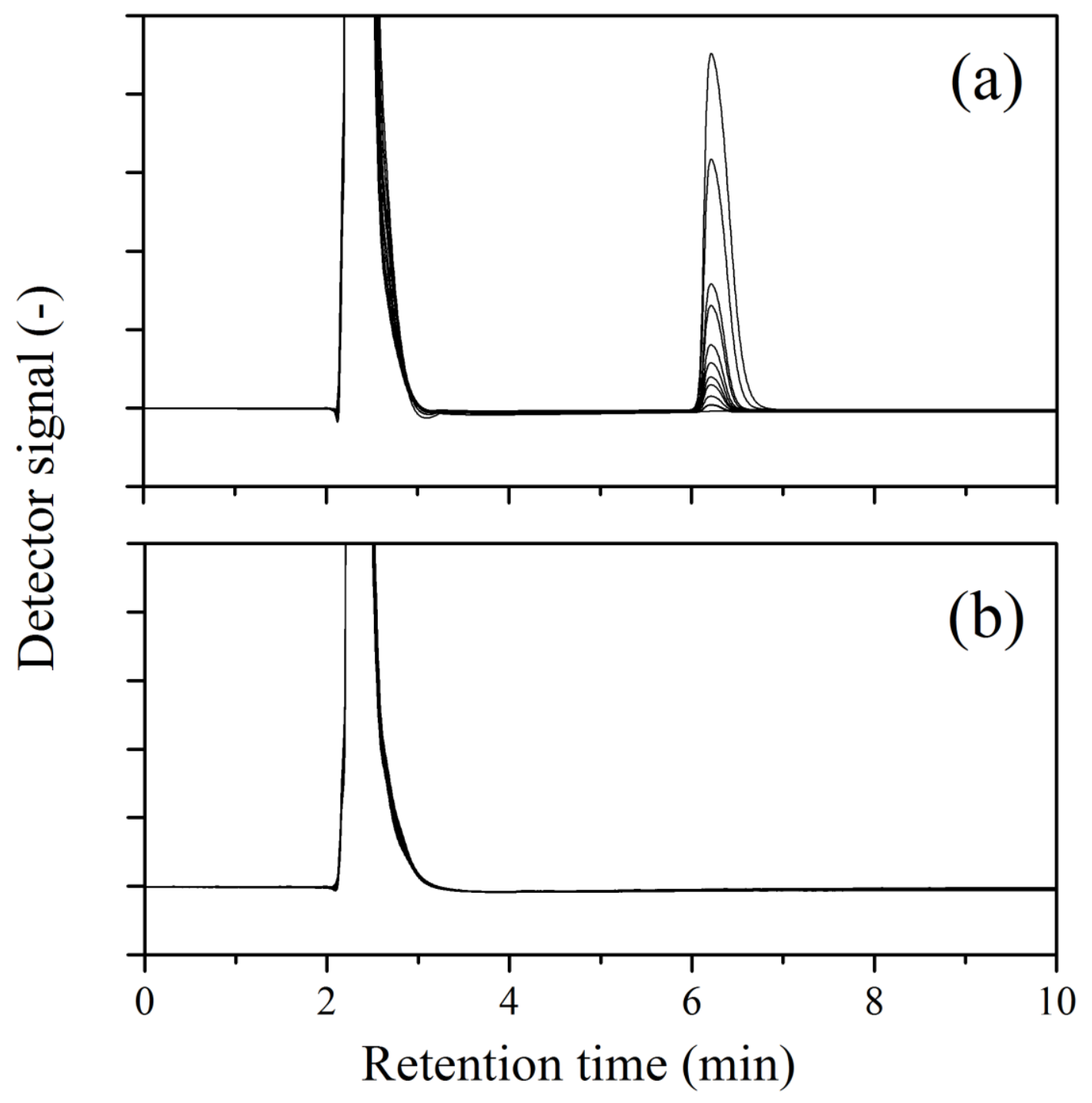


Polyák, Fig. 2

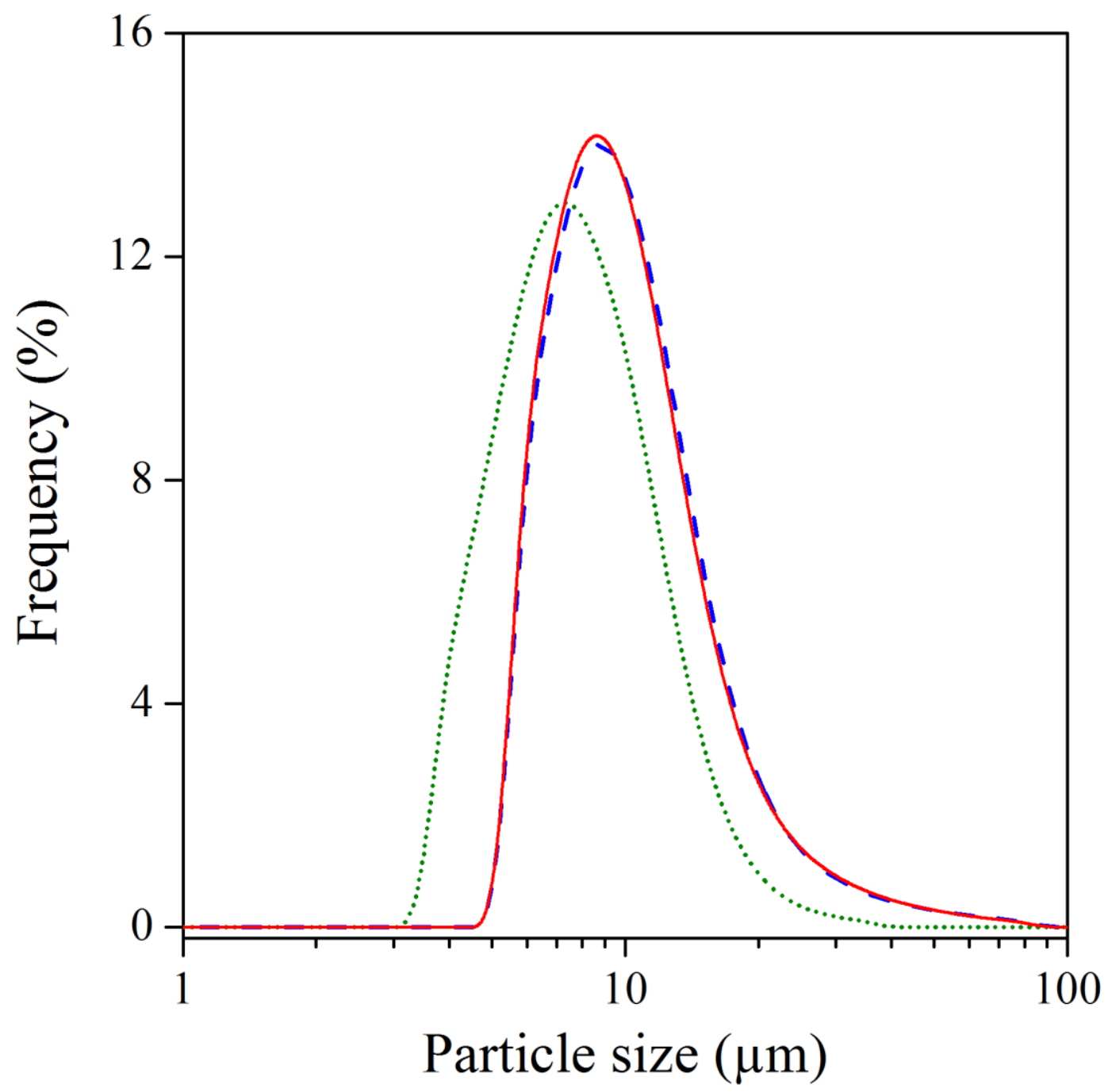


Polyák, Fig. 3

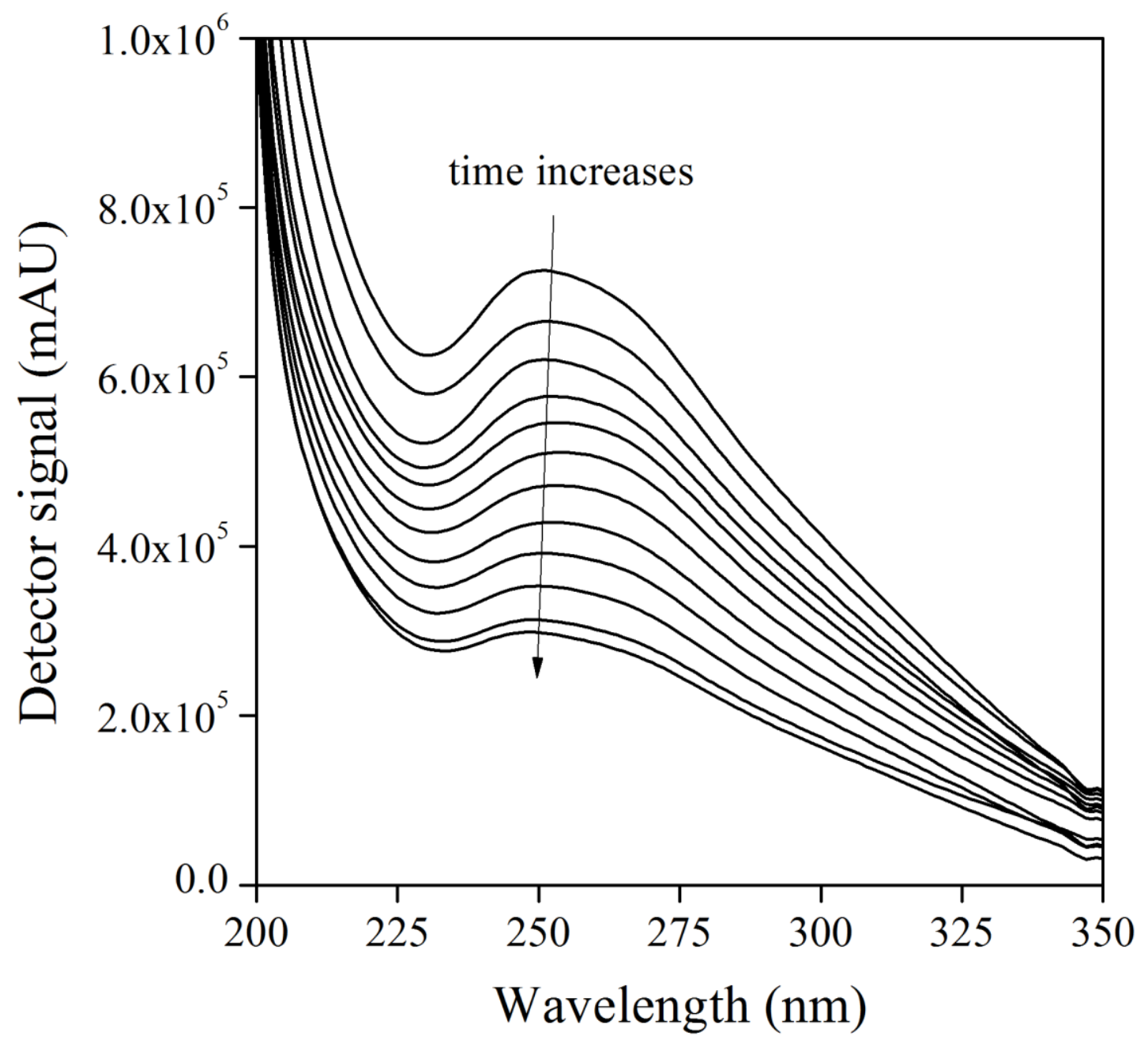


Polyák, Fig. 4

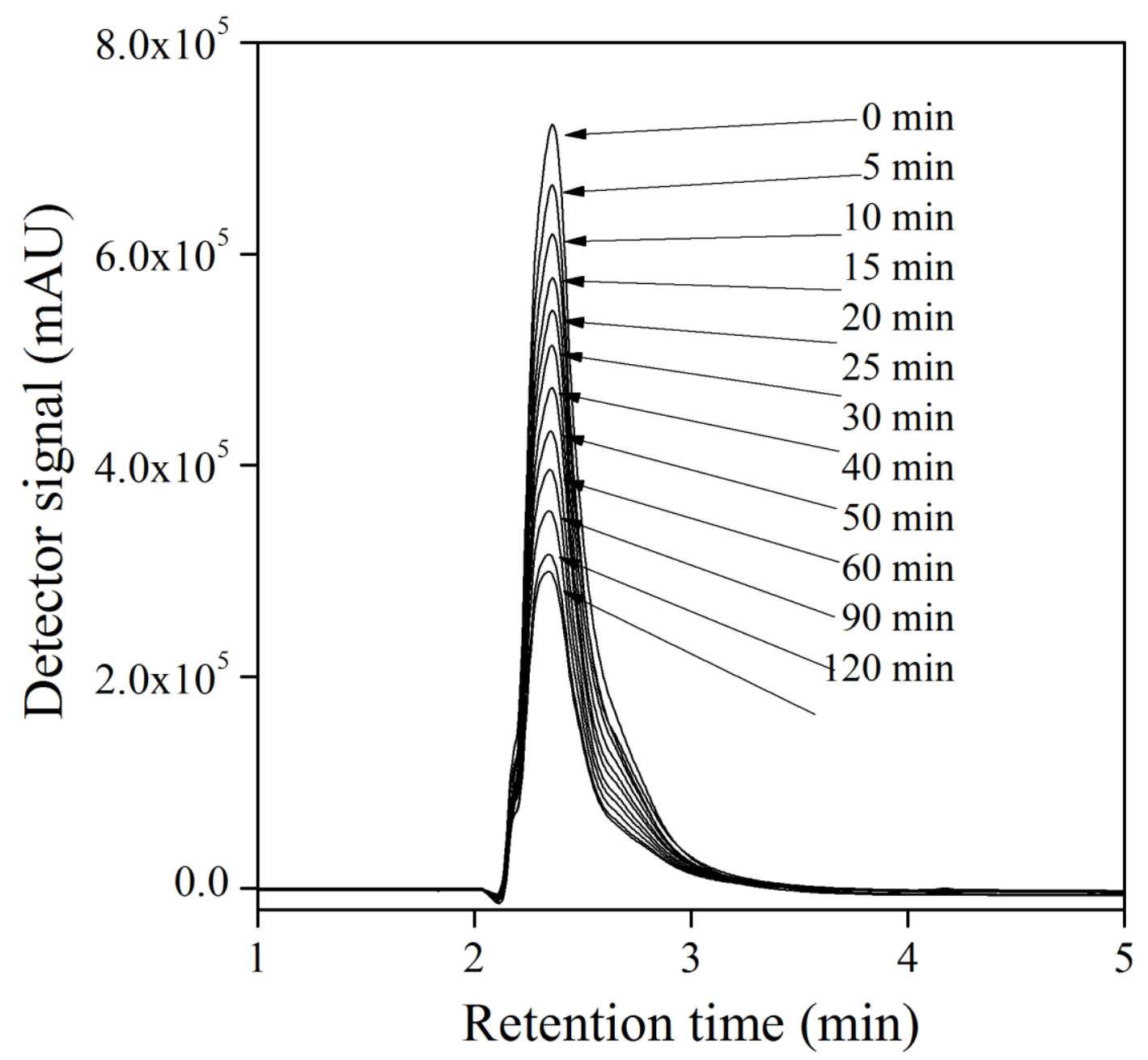


Polyák, Fig. 5

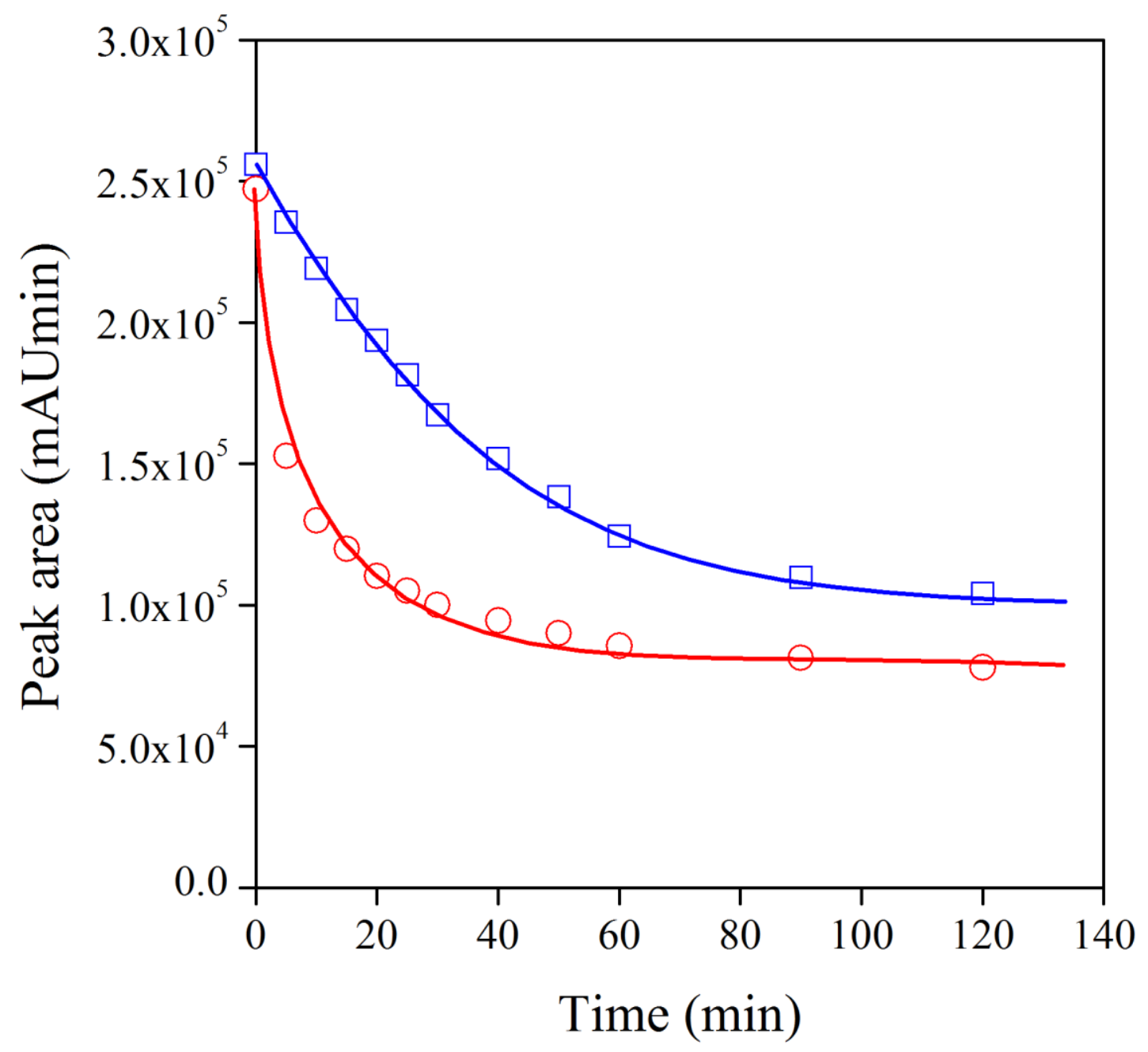


Polyák, Fig. 6

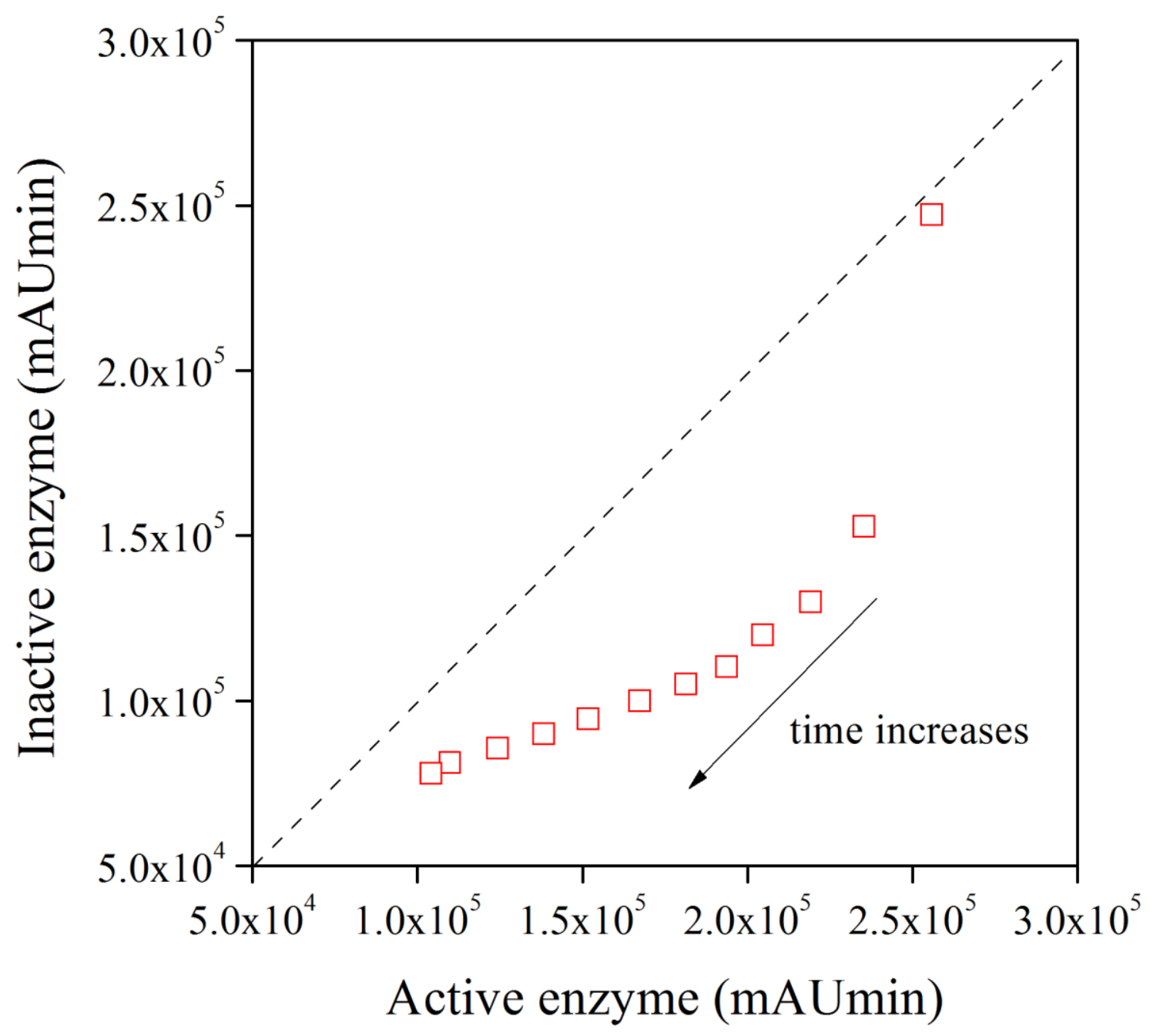


Polyák, Fig. 7

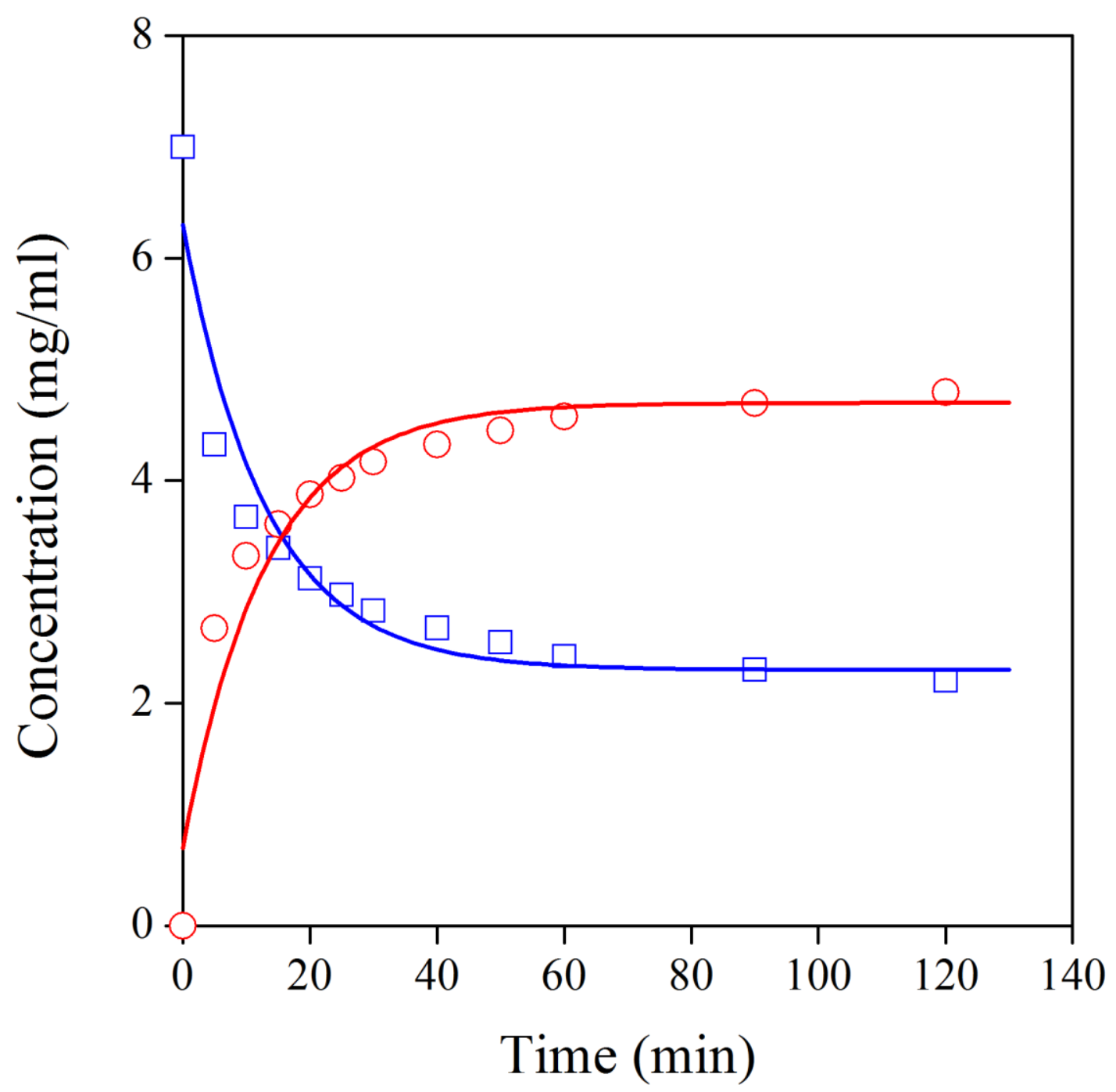


Polyák, Fig. 8

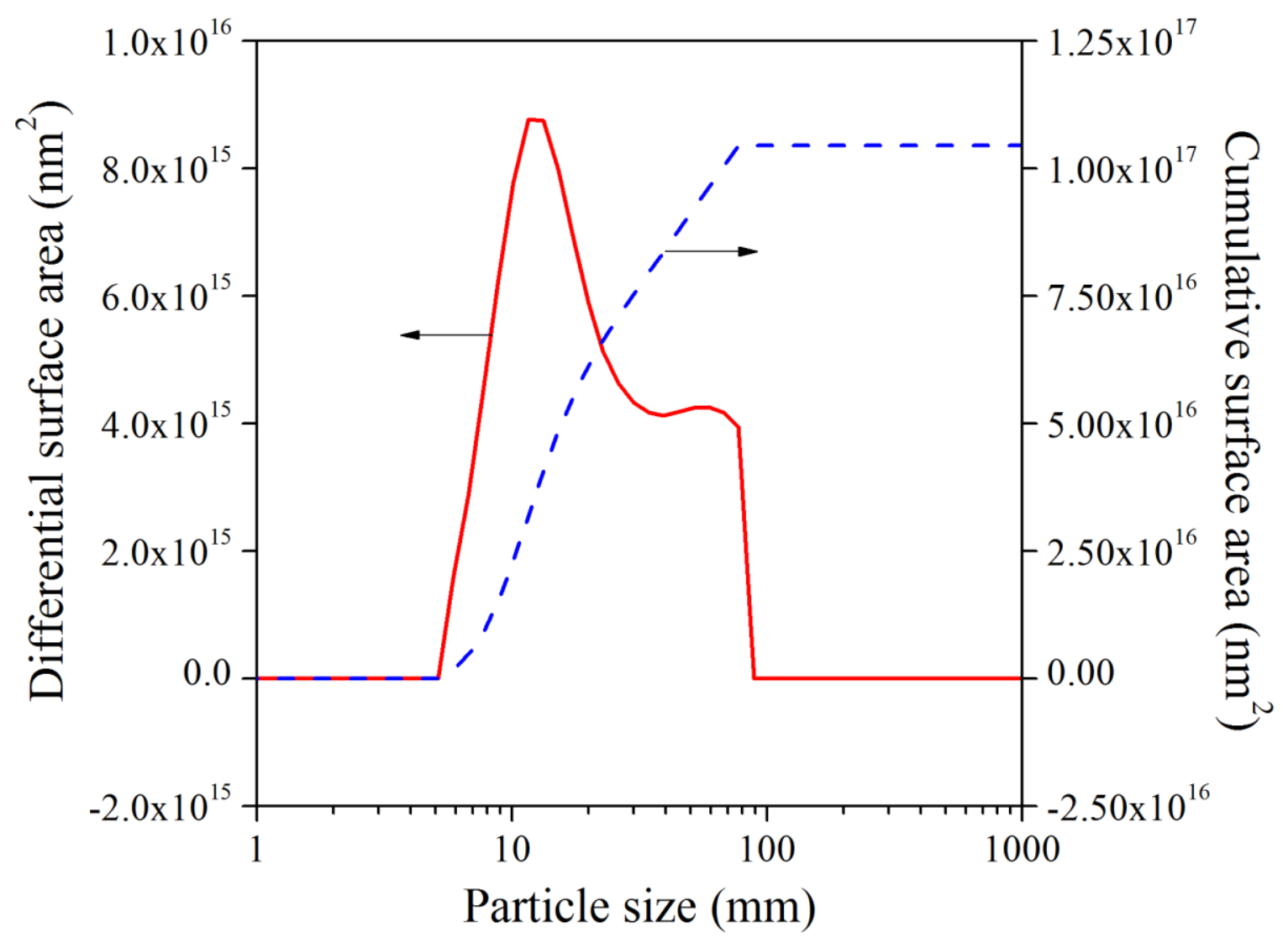

